\title{
The Importance of Research Collaboration for a Postdoctoral Researcher
}

\author{
Akiko Ohata *, Mika Konuma *, Yuji Mizukami ${ }^{\dagger}$
}

\begin{abstract}
To identify the characteristics of optimal research environment for postdoctoral researchers, we examine research papers produced by postdoctoral researchers in relation to their postdoctoral contexts. We find a difference by researcher's environment in papers where the target postdoctoral researcher is not the first author. In particular, the number of papers where the first author belongs to other institutes from the target postdoctoral researchers is quite different. Furthermore, coauthored papers in the postdoctoral researchers are correlated with differences in the H-index, that shows both the productivity and the citation impact of each researcher's publications. This survey by institutional research indicates that collaboration during postdoctoral research may affect the future research career of those researchers.
\end{abstract}

Keywords: H-index, Institutional Research, Postdoctoral researchers, Research career

\section{Introduction}

In Japan, the number of postdoctoral researchers began to increase after the postdoctoral fellows support plan was instituted in 1996 for 10,000 people [1]. Due to the limited number of permanent positions available, finding a permanent position after acquiring a doctorate has long been a matter of serious concern for young researchers. It is a particularly serious concern in Japan, as shown in the fact that the average age of a postdoctoral researcher in Japan is about 35 years, according to an investigation published in 2015 [2]. If students who graduate from university go to work at a company, they will demonstrate an important role by age 35 . Although, in Japan, several studies were conducted on the postdoctoral system of USA, where this role has a long history, the state of postdoctoral researchers and their prospects of finding a good position have not improved at all [3][4]. This indicates that possession of a doctoral degree does not appear to confer an advantage on its bearer, and this led to a significant fall in the number of students at graduate schools. Additionally, this led to a crisis in research at universities, as well as poorer prospects of innovation in industry.

Normally, after a scholar receives a doctorate, the expectation is that research will continue at a university or research institution for several years, as a postdoctoral researcher or in a similar role. During this period, the researcher is expected to seek a more permanent position.

\footnotetext{
* Institute of Space and Astronautical Science, JAXA, Kanagawa, Japan

$\dagger$ College of Industrial Technology, Nihon University, Chiba, Japan
} 
While some do obtain independent research positions, others continue as postdoctoral fellows for long periods. Obtaining a position in industry after being a postdoctoral fellow is rather rare. For this reason, the government has started the Leading Initiative for Excellent Young Researchers program [5]. In this system, new career paths are expected to develop for young researchers, not only in the university context but also in companies. In any case, the optimal environment for postdoctoral researchers has never been adequately established.

We already reported the results of a comparison among research activities in postdoctoral researchers in two countries [6][7]. There, we showed that groups with higher H-index [8] values, that shows both the productivity and the citation impact of each researcher's publications, conduct more research in collaboration with researchers at other institutions. However, the range of postdoctoral researchers examined was limited. In this study, we investigate research activities within a larger group of researchers, namely, those who began between 2010 and 2015 at four positions at different institutions, and we discuss the impact of their collaboration works.

\section{Research Activities of Postdoctoral Researchers at Several In- stitutes}

\subsection{Basic information on the kinds of postdoctoral researchers in this study}

In this study, we compare postdoctoral researchers with three-year term of employment in four groups, as shown in Table 1. Group A demonstrates standard benefits (salary, research funds, etc.), Group B demonstrates standard benefits that are better than those of Group A, and Group C and D demonstrates a high level of benefits. Researchers in Groups A and B demonstrate versions of standard benefits in Japan, and benefits on the level of Groups C and $\mathrm{D}$ are generally found only for high-level postdoctoral researchers. Most researchers in Groups A and B in this study are Japanese. In this study, we only use data for researchers who entered into their postdoctoral positions between 2010 and 2015. The data of researchers for Table 1 were obtained from the following web sites;

https://www.isas.jaxa.jp/about/annual_report/, https://www.riken.jp/pr/publications/spdr/index.html, https://www.stsci.edu/stsci-research/fellowships/nasa-hubble-fellowship-program/2017-and-prior-fellows and https://cxc.harvard.edu/fellows/allFellowsList.html.

All research areas are related to science, excluding such fields as the medical sciences and agriculture-related fields. We use data of papers obtained from the SCOPUS database [9], to assess the subjects' research activities. A coauthored paper is defined as a paper where the target postdoctoral researcher is an author but not the first author.

Table 1: Research positions in this study

\begin{tabular}{|c|c|c|c|c|}
\hline Country & $\begin{array}{c}\text { Reward } \\
\text { (Salary, } \\
\text { Research budget, } \\
\text { etc.) }\end{array}$ & $\begin{array}{c}\text { Contract length } \\
\text { (years) }\end{array}$ & $\begin{array}{c}\text { Number of Ph.D. } \\
\text { between } \\
2010 \text { and 2015 }\end{array}$ \\
\hline Group A & Japan & $\bigcirc$ & 3 & 66 \\
\hline Group B & Japan & $\bigcirc+$ & 3 & 231 \\
\hline Group C & USA & 0 & 3 & 99 \\
\hline Group D & USA & 0 & 3 & 69 \\
\hline
\end{tabular}


Figure 1(a-d) shows the distribution of times when the researchers obtained their postdoctoral positions on which we focused after receiving their Ph.D. Figure 2 shows the H-index values for each researcher. $\mathrm{H}$-index is defined as the maximum value of $\mathrm{H}$, such that the given author has published $\mathrm{H}$ papers that have each been cited at least $\mathrm{H}$ times as shown in Fig. 2(a). It can be seen that the publications of researchers in Groups $\mathrm{C}$ and $\mathrm{D}$ demonstrated higher numbers of citations. Researchers often asserted that a researcher's H-index value grows as their career lengthens, and this tendency is apparent for researchers in Groups $\mathrm{C}$ and D. This sometimes means that researchers in these two groups developed their careers steadily.
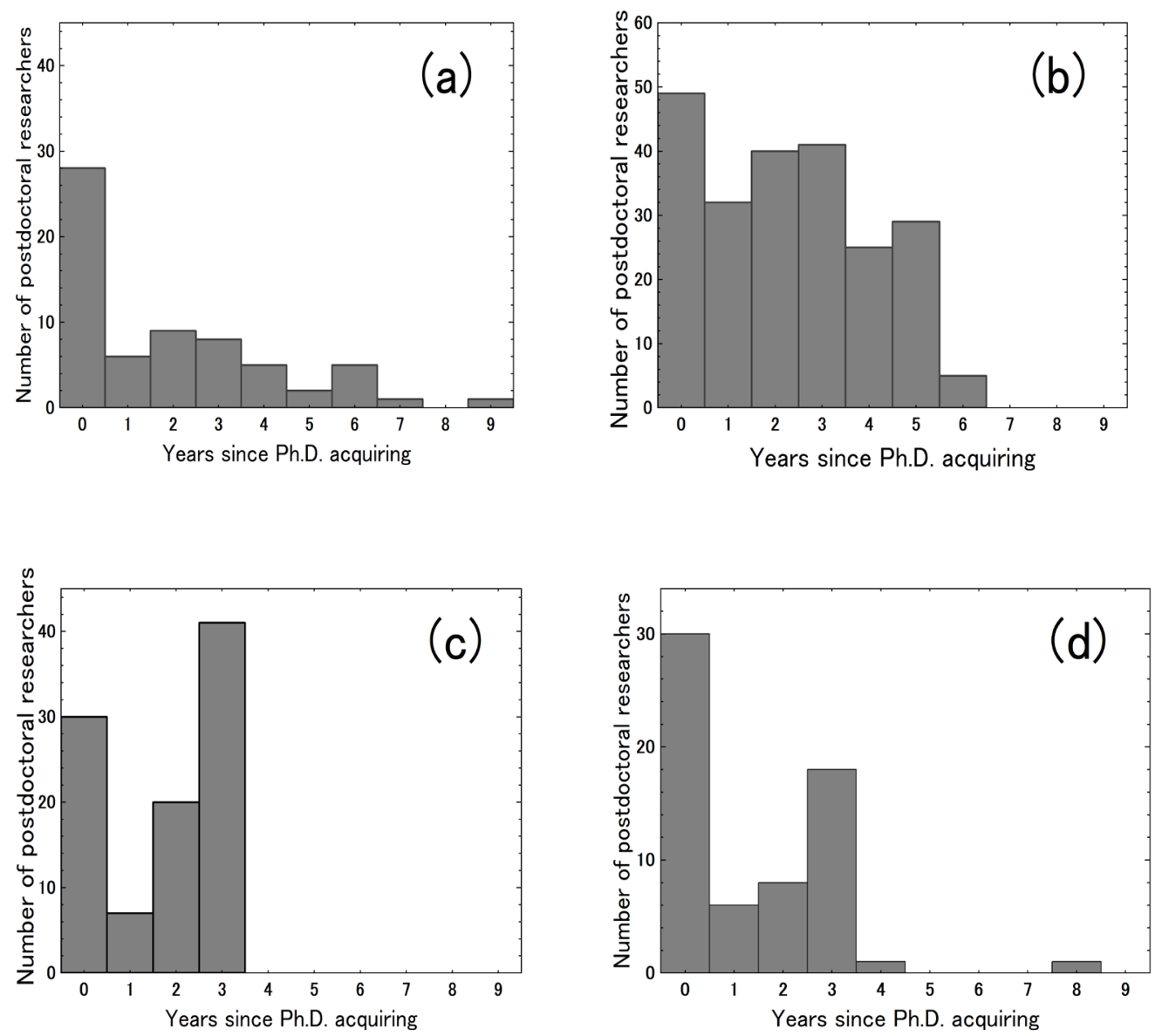

Fig. 1: (a)-(d) Distribution of years since receiving a doctorate for Group A, B, C, and D at the time of finding the given postdoctoral position, respectively. Researchers whose year of receiving their doctorate cannot be determined are not included in these figures. 


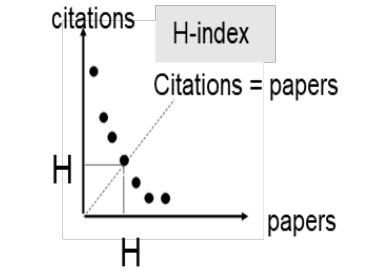

$\mathrm{H}$-index is defined as the maximum value of $\mathrm{H}$ such that the given author has published $\mathrm{H}$ papers that have each been cited at least $\mathrm{H}$ times.

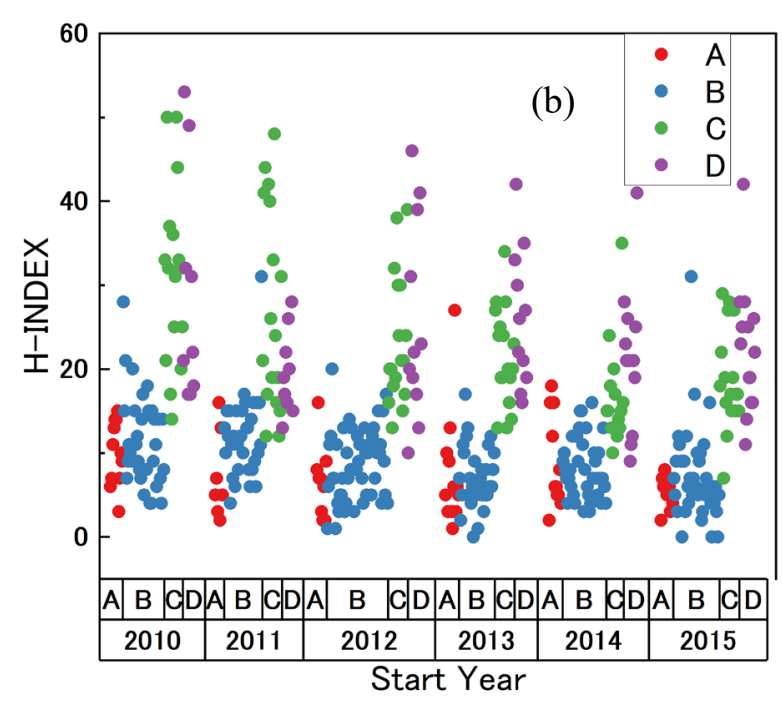

Fig. 2: (a) Explanation of degerming for the H-index. (b) H-index values for each postdoctoral researcher in Groups A, B, C, and D. The Start Year indicates the year when the given researcher began studying at the given postdoctoral position.

\subsection{Publication of research papers}

Figure 3 shows the average values for annual change in number of coauthored and firstauthored papers by the postdoctoral researchers. The results show that the number of papers on which researchers in Groups C and D were contributors as a coauthor increased considerably over the period of interest. Conversely, for the same group, the number of papers with first-author contributions gradually decreased. This suggests that as the researchers in Group $\mathrm{C}$ and $\mathrm{D}$ gradually gained independence, they joined research groups and contributed to research but not as first author.

Next, Figure 4 shows the number of coauthored papers for which the first authors belonged to the same institutions as the postdoctoral researchers. Then, Figure 5 presents the number of first authors from the same or different institutions as the target researcher. The postdoctoral researchers in Group C and D collaborated with more researchers from other institutions. The comparison between the average values for Figures 4 and 5 shows that researchers coauthored papers with many other researchers at different institutions. We already confirmed that even the median values of these parameters do not show the different tendency.

Several differences are found in research conditions for pursing the research between Groups A, B, C, and D, such as the research budget or choosing the research theme. The research budget is provided directly to researchers through their fellowship programs in Groups B, C, and D at a level of $\$ 9,000$ per year for Group B and about $\$ 20,000$ per year for Group C and Group D. Furthermore, the themes pursued by researchers in Groups B, C, and $\mathrm{D}$ are largely determined by the researchers themselves, but in Group A, the research themes 
conform to their institution's requirements. Researchers in this group are only able to spend a certain percentage of their time on themes that match their own interests. Although the research conditions are different between Groups A and B, the number of coauthored papers and the variety of first authorship in the coauthored papers do not differ. Conversely, although the research conditions between $\mathrm{B}, \mathrm{C}$, and $\mathrm{D}$ are rather similar in relation to funding and research theme, the coauthored research papers show rather different numbers.

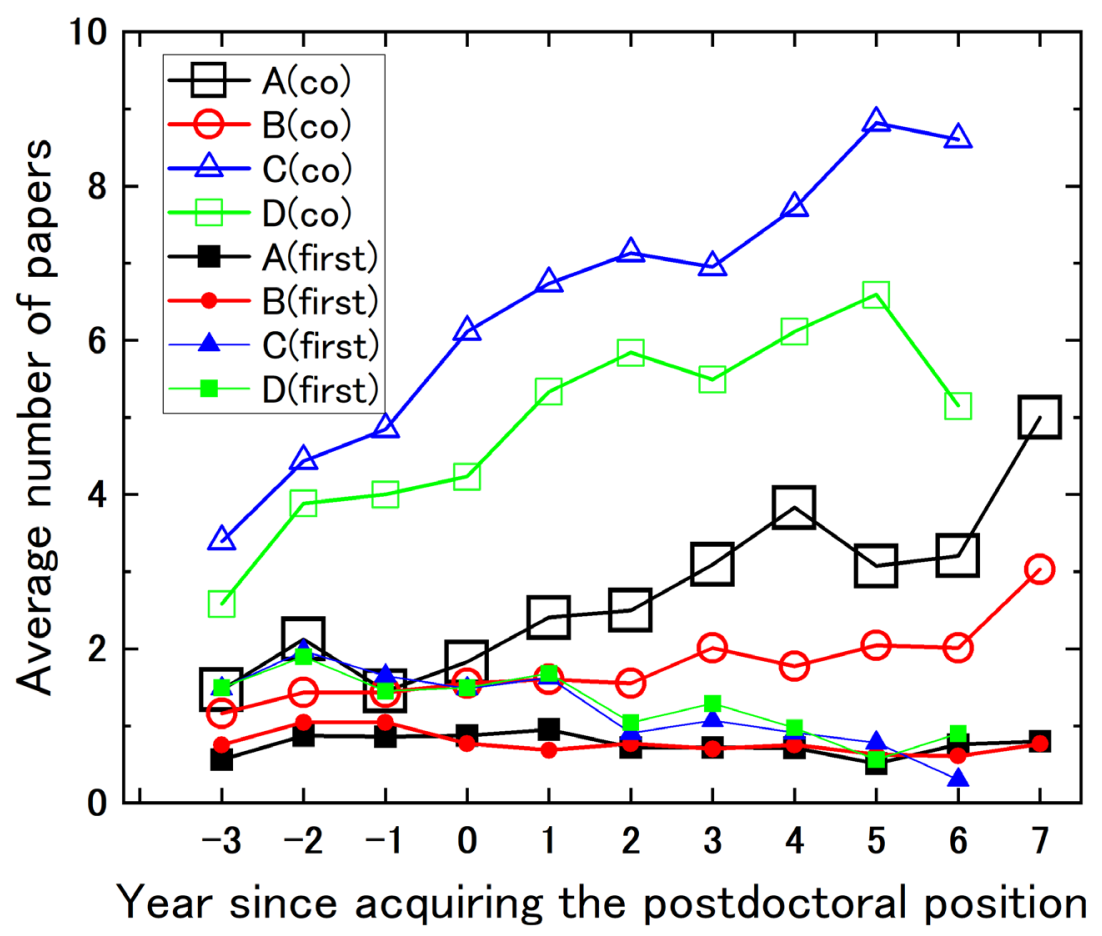

Fig. 3: Average values for number of first-authored papers and coauthored papers for Group A, B, C, and D. The X-axis indicates the year that the researcher is hired for the corresponding postdoctoral position. The notation first in parentheses indicates cases where the given researcher was the first author for a paper, and the notation $c o$ in parentheses indicates cases where the given researcher is not the first author of a paper. 


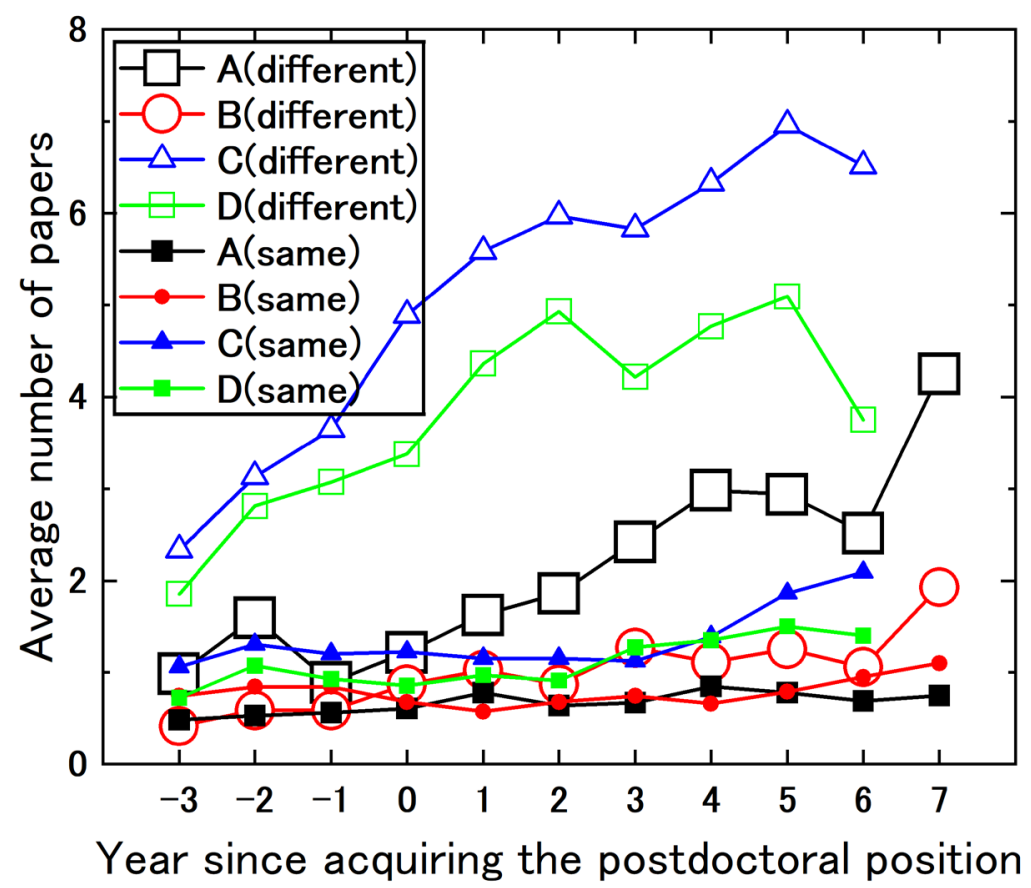

Fig.4: Average values for coauthored papers when the first author is at the same institution as the given researcher or at a different institution for Groups A, B, C, and D.

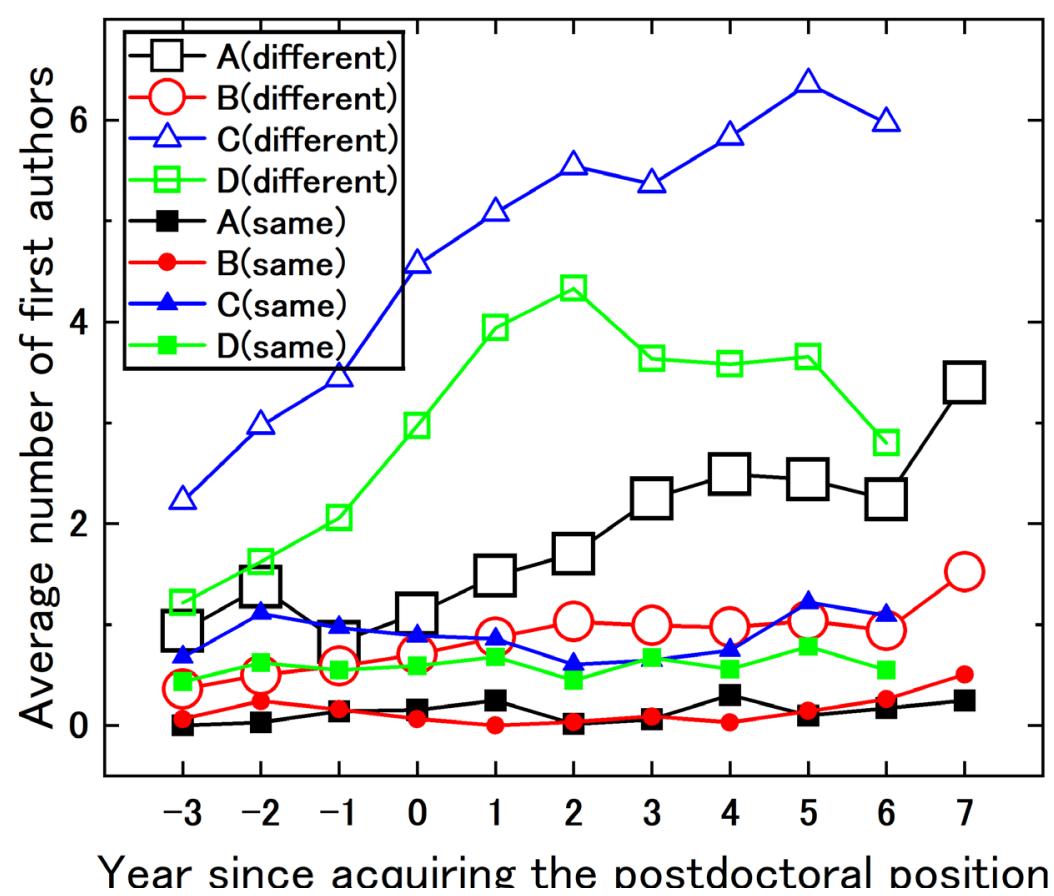

Fig.5: Average values for the number of first authors (omitting duplicates) of coauthored papers with first authors at the same institution as the postdoctoral researchers or at a different one for Groups A, B, C, and D. 


\subsection{Impact of collaboration}

The results given above represent the importance of coauthored research, both with first authors at the institution where the researcher is located and those at other institutions. In this section, we discuss the impact of this collaboration on the citation index.

As noted above, the H-index values for researchers in Groups $\mathrm{C}$ and D were significantly larger than the corresponding values for those in Groups A and B. Figure 6 presents the relationship between the $\mathrm{H}$-index values for all authorship types, including coauthored (H-index all) and first-authored (H-index first author) papers. Next, the straight line indicates cases in which the $\mathrm{H}$-index values obtained from all papers for each researcher $(\mathrm{H}$-index all) are determined only by first-authored papers. In Groups $\mathrm{C}$ and $\mathrm{D}$, the high citation index for collaborative research leads to a high $\mathrm{H}$-index value. For example, for values of $\mathrm{H}$-INDEX $=8$ on the $\mathrm{y}$-axis (H-index first author), the $\mathrm{H}$-index values for all papers (H-index all) are large for Groups $\mathrm{C}$ and $\mathrm{D}$, while the $\mathrm{H}$-index values for all papers (H-index all) are small for Groups A and B. Therefore, collaborative research improved the status of the researchers in Groups $\mathrm{C}$ and $\mathrm{D}$, at least as measured by this index. It is reported that collaborative research work across a wide range of fields can help produce innovation [10][11]. Therefore, the research environment of Groups $\mathrm{C}$ and $\mathrm{D}$ may give rise to breakthroughs.

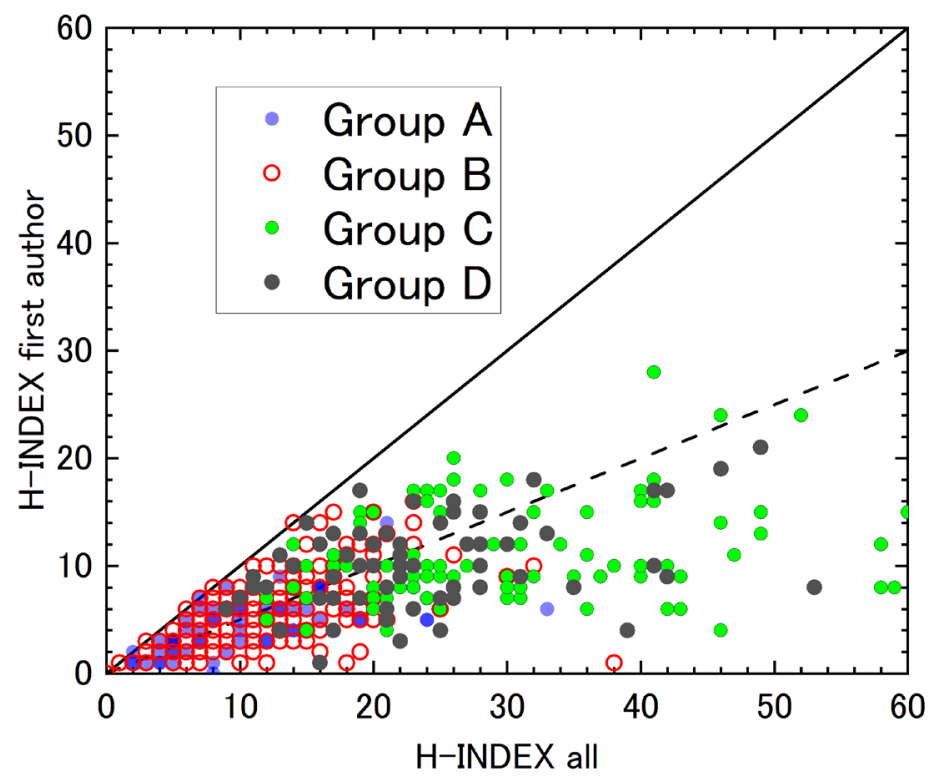

Fig. 6: H-index values obtained from all papers for each researcher (H-index all) vs. Hindex for first-author papers (H-index first author). The straight line presents cases wherein the values for the $\mathrm{H}$-index obtained from all papers for each researcher $(\mathrm{H}$-index all) is determined only by the first-authored papers. The dashed line shows (H-index first author $)=(\mathrm{H}$-index all $) / 2$.

\section{Conclusion}

By investigating research publications in four groups of postdoctoral researchers, we found a difference in the papers they coauthored. The number of papers in which postdoctoral researchers in Group C and D contributed as a coauthor was much larger than those in Group 
A and B. Further, many other researchers exhibited first-author contributions to publications by the postdoctoral researchers in Group C and D. This tendency was particularly apparent for papers that were coauthored with researchers at other institutions.

We also investigated the impact of collaboration on the citation index and found that the high citation index seen for coauthored papers increased the $\mathrm{H}$-index for the given researcher in Groups C and D. As it is reported that collaborative research with a wide variety of researchers can lead to innovation, differences in collaborative research between groups may cause differences in the production of innovative research.

\section{Acknowledgement}

One of the authors (Y. M) is supported by the Research group support project (NU-Innovation). The authors wish to thank K. Hagiwara and Y. Ohshima for helpful discussions and comments on earlier version of this paper.

\section{References}

[1] T. Kobayashi, "What Is the Problem in the Postdoc Issue", Trends in the sciences, Vol.22, No.3, pp.62-63, https://www.jstage.jst.go.jp/article/tits/22/3/22_3_62/_pdf (2017) (In Japanese)

[2] T. Matsuzawa, "Survey for the career path of PhD students: Interest in transferable skills, and awareness of overseas students", http://doi.org/1q0.15108/dp176 (2019) (In Japanese).

[3] M. Okamoto, T. Matsuzawa, T. Inuzuka "The 2015 Survey on Postdoctoral Fellows Regarding Employment and Careers in Japan", http://www.nistep.go.jp/reportlist, http://doi.org/10.15108/rm270 (2018) (In Japanese).

[4] A. Kitano, "Actual conditions and issues of postdoctoral support in the United State", Bulletin of research / Nihon University Faculty of Arts and Sciences Institute of Humanities [ed.] (84): 2012 p.55-70

[5] Japan Society for the Promotion of Science - The Leading Initiative for Excellent Young Researchers (LEADER), https://www.jsps.go.jp/english/e-le/index.html

[6] A. Ohata and K. Hagihara, "Importance of research network analysis for early-career scientists" International Society for Scientometrics and Informatics, Roma, Italy, Sep. (2019), 2, p2700-2701.

[7] A. Ohata, K. Konuma and Y. Mizukami, "What is the Optimal Environment for a Postdoctoral Researcher?" $20209^{\text {th }}$ International Congress on Advanced Applied Informatics (IIAI-AAI) International Society for Scientometrics and Informatics, Kitakyushu, Japan, Sep. (2020), DOI: 10.1109/IIAI-AAI50415.2020.00080. 
[8] Gemma Conroy, "What's wrong with the H-index, according to its inventor", Nature Index, March 24 (2020).

[9] https://www.elsevier.com/solutions/scopus, (The data was accessed in 2019)

[10] A. Iriyama, "World standard management theory", ISBN-10: 4478109575.

[11] M. Baer, "The Strength of Weak Ties Perspective on Creativity: A Comprehensive Examination and Extension”, Journal of Applied Psychology, 95, p592-601. 